D $^{\mathrm{r}}$ J. Krenn. Derzeitiger Stand der Schmirgelmichfrage. Wiener Landw. Ztg., $1932, \mathrm{n}^{\circ} 5$.

Dr Th. Kertesz. Eine bakteriologische Methode zar Verhütung des ,, Ōligwerdens " der Milch. Molk. Ztg. Hildesheim, 1932, $\mathrm{n}^{\circ} 37$.

$D^{r}$ S. KENDE. Weitere Untersuchungen über ölig-talgige ,, schmirgelige ,, Veraenderungen der Milch. Mulchw. Forschungen, 1932, 13, 2-3.

$D^{r}$ G. MAYER. Erfolgreiche Bekaempfung der Schmirgelplage in einem Grossbetrieb. Moltk. Ztg. Hildesheim, 1932, $\mathrm{n}^{\circ} 107$.

Agr. G. Johansson. Ett senare tiders mjölkfel. (Une nouvelle altération du lait). Svenslea Mejeritidningen, 1933, $\mathrm{n}^{\circ} 22$.

$D^{r}$ G. MaJER. Abschliessende praktische Erfahrungen in der Bekaempfung des schmirgeligen, ölig-talgigen Geschmacks der Milch. Molk. Ztg. Hildesheim, $1933, \mathrm{n}^{\circ} 56$.

Dipl. Agr. Hch. HтмmeLsiach. Freiburg : Über das Auftreten des Milehfehlers ,, talgig-tranig “. Südd. Molk. Ztg., 1933, 21-IX.

\title{
LA FABRICATION DES CASÉINES LACTIQUES A FAIBLE TENEUR EN CENDRES
}

\section{par}

JEAN PIEN

Ingénieur-chimiste (I. C. R.),

Docteur ès Sciences, Directeur et

\section{S. HERSCHDOEREER}

Docteur ès Sciences

(Vienne).

des Laboratoires des "Fermiers réunis ".

L'un des éléments les plus significatifs et les plus importants dans l'appréciation de la qualité des caséines lactiques est la teneur en matières minérales. On sait que la caséine lactique théorique ne derrait pas contenir de cendres et que les caséines industrielles les plus recherchées sont celles qui en contiennent le moins.

Après avoir indiqué quelle était la meilleure manière de doser les cendres aveo exactitude (Le Lait, nov. 1933, page 1081) il était dans notre intention d'étudier les facteurs qui, dans la fabrication de la caséine lactique, influencent le plus la teneur en matières minérales de ee produit. Or, Mlle Brigando vient de publier une série d'articles donnant le résultat de ses recherches dans un domaine analogue. Nous avons tenu à retarder la publication de notre travail pour éviter, le cas échéant, un double emploi inutile. Cependant, la récente publication, dans la revue Le Lait, du travail de Mile BRIGANDo (thèse de doctorat d'Université qu'elle a préparée au laboratoire du professeur Ch. Porcher, à Lyon, et soutenue devant la faculté des Sciences de cette ville) nous a finalement incités à publier nos résultats. M ${ }^{11 e}$ BRIGANDO a surtout travaillé la question des caséines d'acidification provoquée alors que nous avions, au contraire, surtout étudié les caséines de fermen- 
tation lactiques spontanée. Il s'agit done de deux domaines légèrement différents.

Nous allons commencer par résumer la théorie la plus vraisemblable, de la formation des cendres dans la préparation des caséines acides ; exposer ensuite des résultats que nous avons obtenus ; tirer enfin des conclusions.

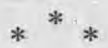

\section{$\S 1$}

\section{THÉORIE DE LA MINÉRALISATION DES CASÉINES ACIDES}

Il paraît évident que la faible minéralisation d'une caséine acide est fonction de la déminéralisation du caséinate phosphocalcique. Cette déminéralisation du caséinate est elle-même fonction de la quantité totale d'acide qui a pu entrer en réaction

D'après la loi des équilibres de membranes de Donnan, nous sommes fondés à admettre que la coagulation de la caséine est un facteur d'arrêt ou du moins de ralentissement pour l'action de l'acide ambiant sur la décalcification du phosphocaséinate déjà occlus dans le précipité. Donc, le résultat le meilleur sera obtenu en faisant réagir la plus grande quantité possible d'acide avant la coagulation : mais on sait que cette coagulation est facilitée par deux facteurs principaux :

a) L'élévation de la température,

b) L'augmentation de l'acidité.

Par conséquent, la meilleure décalcification sera obtenue par des acidifications à froid, d'une part; d'autre part, par des acidifications non susceptibles d'entraîner des coagulations prématurées.

Cette dernière condition ne sera pas réalisée si on introduit un acide insuffisamment dilué qui détermine au point de chute une acidité très élevée et un eaillage ponctuel générateur de précipités incomplètement décalcifiés. La condition sera au contraire assez bien réalisée par des apports très lents, d'acide très dilué dans du lait violemment agité (technique de VAN SLYKE). Enfin cette condition sera idéalement réalisée par la fermentation lactique qui est lente, présente en tous points de la masse et très progressive.

Done : l'acidification à froid et dans des conditions se rapprochant de celles de fermentations lactiques spontanées, est celle qui, a priori, conduira aux meilleurs résultats au point de vue taux de cendres.

Il semblerait done que la question de la température doive jouer un rôle prépondérant. Il en est bien ainsi en fait. Mais la cause véritable de la plus ou moins grande décalcification n'est pas la température, mais bien la quantité d'acide qui aura pu réagir avant 
coagulation. Cette quantité étant elle-même directement influencée par la plus ou moins grande précocité de la coagulation et, indirectement, par la température qui conditionne, à son tour, cette précocité. Dans l'ordre d'importance décroissante, nous classerons done les facteurs de la décalcification de la façon suivante :

$1^{\circ}$ d'abord la quantité d'acide qui a pu entrer en réaction ; $2^{\circ}$ ensuite la plus ou moins grande précocité de la coagulation (qui limite le rôle du facteur no 1 ) ;

$3^{\circ}$ enfin la température (qui détermine le facteur $n^{\circ} 2$ ).

Remarque. - Quand nous parlons de quantité d'acide produit ou introduit, nous n'excluons nullement la notion de concentration d'acidité (potentielle ou actuelle), car il est probable que ces deux notions doivent intervenir simultanément. C'est même à cette seconde notion exclusivement, que nous faisons appel dans notre travail sur la coagulation lactique (concentration d'acide et concentration d'ions $\mathrm{H}$ ). Il ressort de cette manière de voir que, pour nous, le point fondamental de l'étude de la décalcification sera le $p \mathrm{H}$ et la concentration d'acide immédiatement avant la coagulation (réalisée à froid ou à chaud) et a fortiori avant la cuisson pour le cas des caséines précipitables à froid. Il convient de noter aussi que l'acidification du sérum pourra se poursuivre après coagulation sans qu'il en résulte une décalcification plus poussée. Et en aucun cas, l'acidité finale après coagulation ne doit entrer en ligne de compte pour juger de la décalcification obtenue.

\section{** \\ $\S 2$ \\ ESSAIS EFFECTUES SUR LES CASÉINES LACTIQUES D'ACIDIFICATION SPONTANÉE.}

I. PRINcipe de L'Étude. - Nous avons tenu à nous placer dans les conditions du travail industriel, c'est-à-dire : fermentation lactique spontanée à la température ordinaire, suivie d'une cuisson dans le sérum à une température toujours au moins égale à $50^{\circ}$. Nous avons volontairement éliminé l'inconnue du lavage en ayant soin dans tous nos essais de laver la caillebotte à fond (trois lavages à l'eau ordinaire et deux lavages à l'eau distillée, entrecoupés de pressages).

Notre seule variable a été en principe l'acidité du lait au moment de la cuisson (acidité obtenue par fermentation à froid). En réalité, cette variable s'est nécessairement accompagnée d'un autre facteur : la température à laquelle le lait a dû être porté pour obtenir la précipitation, dans le cas où celle-ci n'avait pas eu lieu à la température ordinaire (cas de faibles acidités). C'est donc l'influence, sur le 
taux de cendres, de l'acidité du lait au moment du chauffage, que nous avons étudiée. Accessoirement nous avons noté l'influence de diverses températures de cuisson sur les caillebottes précipitées à froid.

II. TEснnique. - Du lait écrémé est abandonné à $20^{\circ}$ à la fermentation lactique spontanée. Chaque essai est effectué sur 5 litres de lait. La fermentation est poursuivie pendant des temps variables correspondant à des acidités différentes. L'acidité est évaluée à l'acidimètre Dornic. Le $p H$ électrométrique du lait (caillé ou non) est également déterminé.

Si le lait est caillé, nous cuisons la caillebotte dans son sérum à $50^{\circ}$ pendant 30 minutes (et dans certains cas à des différentes températures pour une même caillebotte).

Si le lait n'est pas caillé, nous le chauffons pour déterminer la précipitation de la caillebotte et nous cuisons celle-ci en maintenant la température de précipitation pendant 30 minutes.

Après cuisson, l'acidité du sérum est notée, la caillebotte est lavée trois fois à l'eau de ville, deux fois à l'eau distillée en ayant soin de la presser fortement entre chaque lavage. Elle est finalement séchée entre 80 et $100^{\circ}$ (rappelons que nous n'avions en vue que l'étude de la teneur en matières minérales).

Les cendres sont déterminées par la méthode Shaw modifiée que nous avons exposée.

III. RÉSULtATS OBTENUS. - Tous nos résultats sont rassemblés dans le tableau suivant :

\begin{tabular}{|c|c|c|c|c|c|c|c|}
\hline \multirow{3}{*}{$\begin{array}{c}\text { Noa } \\
\text { des } \\
\text { essesis }\end{array}$} & \multirow{2}{*}{\multicolumn{2}{|c|}{$\begin{array}{l}\text { Caractères du } \\
\text { lait au } \\
\text { moment du } \\
\text { chauffage }\end{array}$}} & \multicolumn{2}{|c|}{ Températures } & \multirow{3}{*}{\begin{tabular}{|} 
Acidité \\
du \\
sérum
\end{tabular}} & \multirow{3}{*}{$\mid \begin{array}{c}\text { Cen- } \\
\text { dres de } \\
\text { la } \\
\text { caséine } \\
\text { lavée } \\
\text { et } \\
\text { séchée }\end{array}$} & \multirow{3}{*}{ Observations } \\
\hline & & & \multirow{2}{*}{$\begin{array}{c}\text { de la précipi- } \\
\text { tation }\end{array}$} & \multirow{2}{*}{ de la cuisson } & & & \\
\hline & $p$ H & $\begin{array}{l}\text { acidité } \\
\text { Dornie }\end{array}$ & & & & & \\
\hline $1 \ldots$ & 6,02 & 27 & 85 & 85 & 18 & 5,47 & \\
\hline $2 \ldots$ & 6,01 & 30 & 80 & 80 & 18 & 5,10 & \\
\hline $3 \ldots$ & 5,76 & 35 & 80 & 80 & 23,5 & 3,84 & \\
\hline $4 \ldots$ & 5,34 & 45 & 80 & 80 & 32 & 3,97 & \\
\hline $5 \ldots$ & 5,23 & 46 & 70 & 70 & 37 & 2,39 & caillé très élastique, fusible à chaud \\
\hline 6... & - & 64 & 50 & 50 & 46 & 0,56 & eaillé élastique \\
\hline $7 \ldots$ & 4,54 & 72 & caillé à froid & 100 & 60 & 0,51 & caséine jaune \\
\hline $8 \ldots$ & 4,54 & 72 & n & 60 & 60 & 0,50 & caséine jaune faihle \\
\hline $9 \ldots$ & 4,54 & 72 & $n$ & 50 & 60 & 0,36 & caséine à peine jaune, bon aspect. \\
\hline $10 \ldots$ & 4,54 & 75 & " & 70 & - & 0,47 & easéine bla ne mat \\
\hline $11 \ldots$ & 4,54 & 75 & $n$ & 37 & - & 0,44 & caséine bla nche brilla nte \\
\hline $12 \ldots$ & 4,30 & - & 》 & 50 & 52 & 0,47 & caséine blanc mat, bon aspect \\
\hline $18 \ldots$ & 4,40 & 85 & $n$ & 50 & 64 & 0,37 & easéine bla nche, bon aspect \\
\hline $14 .$. & - & 86 & » & 50 & 68 & 0,25 & caséine bla nche, très bon aspect \\
\hline
\end{tabular}


Les résultats contenus dans ce tableau sont traduits dans les graphiques qui accompagnent l'article.

IV. commentaires. - Ces résultats appellent les commentaires suivants :

$1^{\circ} \mathrm{Il}$ existe des caséines lactiques riches ou très riches en cendres, malgré d'excellents lavages.

$2^{\circ}$ Il y a un rapport étroit entre la teneur en cendres d'une caséine bien lavée et l'acidité du lait au moment où on l'a soumis à l'action de la chaleur.

Plus l'acidité atteinte, à froid par le lait, est élevée, plus la teneur en cendres de la caséine est faible.

GRAPHIQUE I.

\section{INFLUEMCE DU PH AU MOMENT DE LA CUISSON SUR LE TAUX DES CENDRES}

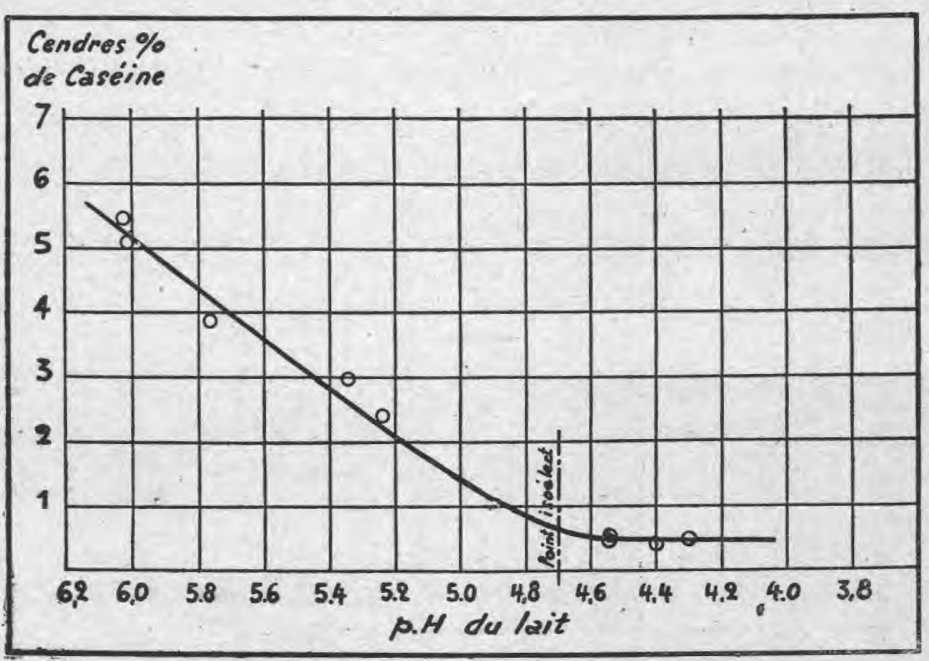

Plus l'acidité atteinte à froid est faible, plus il faut chauffer pour obtenir la précipitation et plus la teneur en cendres est élevée. On pourrait admettre avec $\mathrm{M}^{\mathrm{Hl}}$ BRIG ANDo, que dans ce dernier cas, e'est l'excès de température qui est cause de l'excès de cendres. En réalité, dans le cas de la caséine lactique spontanée, ces deux phénomènes: acidité et température de précipitation, sont liés (comıme nous l'avons indiqué plus haut) et il nous paraît plus logique de penser que c'est l'insuffisance de l'acidité qui nuit à une bonne décalcification du caséinate. 
GRAPHIQUE II,

\section{IMFLUENCE DE L'ACIOITÉ AUS MOMENT OE LA CUISSON SUR LE TAUX DES CENORES}

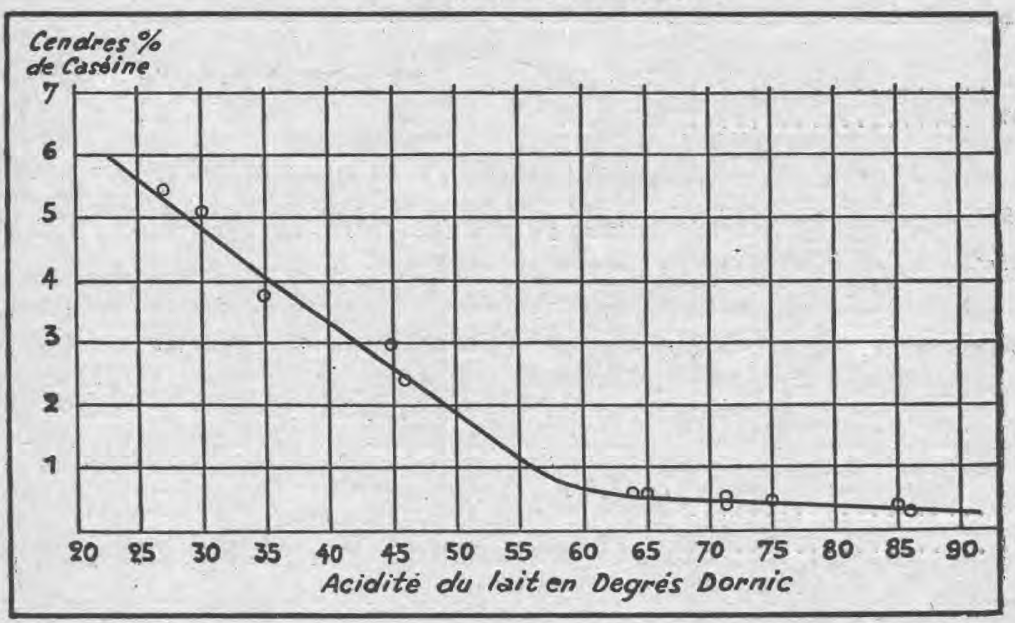

$3^{\circ}$ Toutes les caillebottes entièrement précipitées à froid (et bien lavées) sont pauvres ou très pauvres en cendres.

40 La température de cuisson d'une caillebotte précipitée à froid est sans grande influence sur la teneur en cendres (essais n $\mathrm{n}^{\mathrm{os}} \mathbf{7}$ à 11). Toutefois, les températures les plus faibles $\left(50^{\circ}\right)$ sont plus favorables à un bon lavage du caillé.

$5^{\circ}$ Comme il était facile de le prévoir, les caillebottes entièrement précipitées à froid au-dessous du point isoélectrique sont les plus pauvres en cendres. Le titrage acidimétrique pour la pratique courante renseigne assez bien sur ce point et permet d'éviter les titrages potentiométriques. L'acidité minimum à obtenir avant la cuisson paraît être de 65 à $70^{\circ}$ Dornic.

$6^{\circ}$ Toutes nos caséines ont été séchées à $80-100^{\circ}$ (après avoir été parfaitement lavées). Cette température relativement élevée ne semble done pas avoir par elle-même d'inconvénients graves pour la teneur en cendres, ni même pour l'aspect du produit si les autres conditions de la fabrication ont été menées de façon satisfaisante.

v. remaroue. - A titre de comparaison nous avons également effectué quelques essais concernant les caséines chlorhydriques. Les résultats obtenus sont très voisins de ceux publiés par Mile BRIgando :

10 A égalité de $p \mathrm{H}$ les précipitations effectuées à des températures différentes entraînent des écarts de cendres considérables : 


\begin{tabular}{|c|c|c|c|c|}
\hline Nos des essais. & $p$ H du sérum & $\begin{array}{c}\text { Température } \\
\text { de la } \\
\text { précipitation }\end{array}$ & $\begin{array}{c}\text { Température } \\
\text { de la } \\
\text { cuisson }\end{array}$ & $\begin{array}{l}\text { Cendres de la } \\
\text { caséine lavée à } \\
\text { fond et séchée }\end{array}$ \\
\hline $15, \ldots \ldots \ldots \ldots \ldots \ldots$, & 5,00 & $36^{\circ}$ & $36^{\circ}$ & 0,40 \\
\hline $16 \quad \ldots \ldots \ldots \ldots \ldots \ldots$ & 4,96 & $50^{\circ}$ & $50^{\circ}$ & 3,36 \\
\hline
\end{tabular}

$2^{\circ}$ A égalité de température de précipitation, les différences d'acidité influencent également la teneur en cendres :

\begin{tabular}{|c|c|c|c|c|}
\hline Nos des essais & $p H$ du sérum & $\begin{array}{c}\text { Température } \\
\text { de la } \\
\text { précipitation }\end{array}$ & $\begin{array}{c}\text { Tempórature } \\
\text { de la } \\
\text { cuisson }\end{array}$ & $\begin{array}{l}\text { Cendres de la } \\
\text { caséine lavée à } \\
\text { fond et sérhéo }\end{array}$ \\
\hline $16 \ldots \ldots \ldots \ldots \ldots \ldots \ldots$ & 4,96 & $50^{\circ}$ & $50^{\circ}$ & 3,36 \\
\hline $17 \ldots \ldots \ldots \ldots \ldots \ldots$ & 4,31 & $50^{\circ}$ & $50^{\circ}$ & 2,74 \\
\hline
\end{tabular}

3 . Notons encore l'importance qui s'attache à des dosages de cendres aussi exacts que possible.

GRAPHIQUE III.

INFLUENCE OE LA TEMPÉRATURE OE CUISSON SUR LE TAUX DE CENDRES

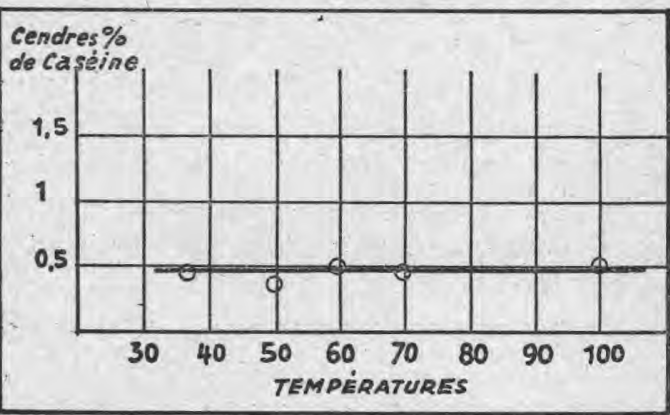

Nous effectuons désormais tous nos dosages de matières minérales par la méthode que nous avons exposée (Le Lait, nov. 1933 , p. 1081). Si nous reprenons les chiffres donnés par MHe BRIGANDO dans son tableau XXIV (Le Lait, sept-oct. 1933 , p. 989), nous voyons que pour des $p \mathrm{H}$ de précipitation variant de 4,0 à 5,0 les cendres qu'elle obtient varient de 0,24 à 2,22 (rapport de 1 à 10). Si les cendres dans ce cas particulier avaient été effectuées par la méthode Shaw modifiée, les chiffres auraient été de l'ordre de 0,2 à 0,6 (rapport de 1 à 3 ).

$\mathrm{Si}, \mathrm{d}$ 'autre part, la comparaison est faite avec les essais analogues effectués sur les caséines lactiques, on s'aperçoit que, passant d'un $p \mathrm{H} 4$ à un $p \mathrm{H} 5$ pour une même température, nos cendres passent de 0,4 à 1,4 (rapport de 1 à 3 ), e'est-à-dire même taux de variation que sur les caséines chlorhydriques, ce qui se eonçoit. 
Mais cette analogie n'apparaîtrait pas si les cendres étaient effeetuées par une méthode inexacte ou à plus forte raison par des méthodes différentes.

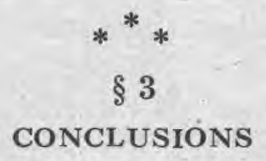

Les conclusions qui se dégagent de l'étude de la fabrication des caséines lactiques par fermentation spontanée, en ce qui concerne uniquement leur teneur en matières minérales, sont les suivantes: le minimum de cendres sera obtenu par une fermentation entièrement conduite à froid jusqu'à un taux d'acidité devant toujours dépasser $65-70^{\circ} \mathrm{D}$.

La cuisson peut être faite à n'importe quelle température, mais il est avantageux pour l'efficacité du lavage de la conduire à la température la plus basse, compatible avec le souci de ne pas perdre la matière par émiettement.

Les conditions de température de fermentation et d'acidité finale étant respectées, la diminution du taux des cendres sera uniquement fonction de la perfection des lavages. Ceux-ci, pour être très efficaces, devraient être séparés par des pressages et effectués avec l'eau aussi pure que possible.

\section{UNE NOUVELLE MÉTHODE DE NUMÉRATION DIRECTE DES MICROBES DULAIT(1)}

(Méthode de Dreyer-Koroleff)

par

\section{W. M. BOGDANOFF}

Docent en Bactériologie, Institut de Laiterie à Wologda (Russie du Nord).

Les méthodes de numération de Ja microflore - celle des plaques et celle dite "de numération directe" - qui existent aujourd'hui, possèdent des avantages et des inconvénients.

La première ne peut être remplacée quand la préparation doit contenir des cellules vivantes et des cellules mortes. Cependant la

(1) Ce travail constitue véritablement une citation rétrospective du travail du défunt professeur S. A. Konolefr, de l'Institut de Laiterie à Wologda : "Nouvelle méthodo do numération directe des cellules dans le plan total du comptage microbiologique n.

La méthode décrite a trouvé une large application dans la pratique des travaux des Instituts scientifiques expérimentaux de I'U. R. S. S.

Nous espérons, par la publication de cet articlø, obtenir une critique de la méthode préconisée. 